\title{
Design of the positioning device for the volatile crowd based on the BD satellite
}

\author{
Zhonghu Yuan ${ }^{1, a^{*}}$, Zhimin Zhang ${ }^{2, b}$, Xiaowei Han ${ }^{3, c}$, Dan Liu ${ }^{4, d}$ \\ 1,2,3,4 The College of Information Engineering, Shenyang University, Shenyang,China \\ asyyzh62@163.com, b15524312389@163.com, chxw69@163.com, ${ }^{\mathrm{d}} 1401771214 @ q q . c o m$
}

\begin{abstract}
Keywords: positioning system, Beidou Satellite, GSM, wireless communication
Abstract: This paper presents design ideas based on BD system of a volatile crowd positioning device by analyzing the shortcomings of the traditional positioning device. The terminal module of this esign uses the STC11F04E as the micro processing chip and the SIM900A module as the wireless communication module, and positioning device was based on BD positioning system of the third generation UM220-III-N double system chip for accurate and stable positioning. The control software was based on the Android system development application software.Test results show that the positioning device can activate the terminal equipment by sending text messages to obtain the location of the terminal equipment, which is displayed on the map.
\end{abstract}

\section{The introduction}

Incomplete statistics missing children every year China has about 20 million and not looking back to less than $0.1 \%$ according to Chinese news reports from the different statistical data.China over the age of 60 about $58 \%$ people with varying degrees of Alzheimer's disease, which led to the annual rate of the elderly lost more frightening, and reaching about 76 million people.

The traditional solution has little effect in the face of the social problems that need to be solved.The using of high technology to solve the problem of life and society has become a fashion in the development of the science and technology. At present, there are some positioning products in the domestic and foreign markets, but the disadvantage is that most of the positioning equipment is based on the GPS positioning system, which has not been used in China's own research and development of the BD Positioning System[1]: power consumption is very large and not conducive to the using of foreign minister's time as a hand-held device;the price is more expensive and function and operation are very complex. It's very meaningful to develop a low power consumption, low price, function and simple operation of positioning equipment based on these shortcomings.

The terminal module of this design uses the STC11F04E SCM as the micro processing chip, and power consumption is low, which supports remote communication and wake up.SIM900A module based on GSM network system is a wireless communication module[2]. Based on the BD Positioning System of the third generation BD chip UM220-III-N dual system chip for accurate and stable positioning.The control software is based on the Android system development application software, which access the address command by sending a text message, the return address data show in map.

\section{Structure of terminal module system}

The design is based on STC11F04E as MPU, which including several parts of the peripheral module:LM2941 power module,SIM900A module and UM220-III-N dual system module.Fig.1 shows the system structure diagram:STC11F04E microprocessor as the core processing module of the whole monitor is responsible for sending and receiving short message command, and receiving the command to parse and execute the command, and sending and receiving compass module command and data, and processing data to obtain the latitude and longitude information;the power module is responsible for the power supply of the whole module, and provides a stable DC power supply for each module;the SIM900A module is responsible for the communication between the controller and the single chip microcomputer SMS;UM220-III-N dual system module is responsible 
for obtaining the current position of the latitude and longitude and transmitting the geographic information to the MPU.

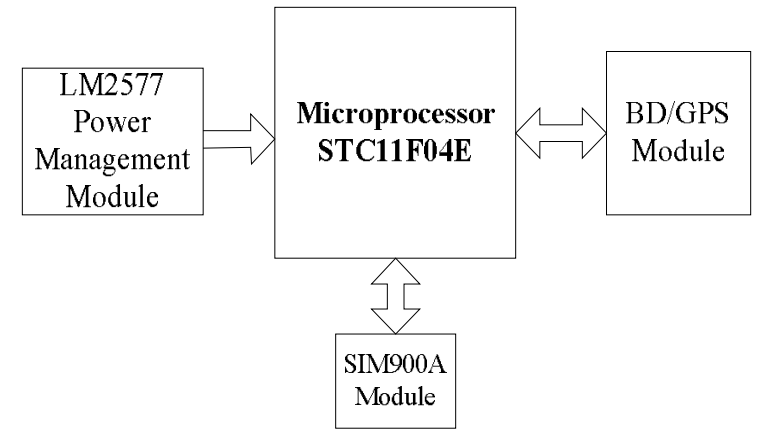

Fig.1

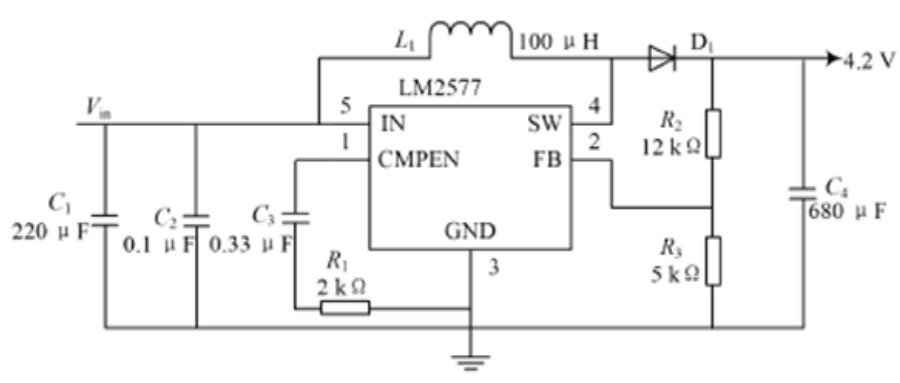

Fig. 2

\section{Hardware design}

\section{Power module design}

The power module design is very important for the normal operation of the module and the module has three modules need to consider the specific power design.SIM900A uses 3.4V to 4.5V for VBAT single supply power supply.This design uses ordinary mobile phone battery power supply,which the battery voltage is generally $3.6 \sim 4.0 \mathrm{~V}$, and we can choose LM2577 power chip.LM2577 can stabilize the battery voltage in the $4.2 \mathrm{~V}$, and can output the maximum current $3 \mathrm{~A}$ at the same time in order to ensure the peak current consumption.Schematic diagram of power supply circuit is shown in Fig. 2.

The UM220-III-N module needs to design the power supply circuit separately in order to avoid the voltage drop caused by the SIM900A module and the module is shut down and the requirements of the other modules to the voltage are required.The voltage range of UM220-III-N is DC: $+2.7 \sim 3.3 \mathrm{~V}$ and its power consumption is $120 \mathrm{~mW}$.Using LM317 adjustable voltage regulator device and its output voltage is $3.3 \mathrm{~V}$ to the requirement of power supply design without too much .UM220-III-N power supply circuit as shown in Fig. 3

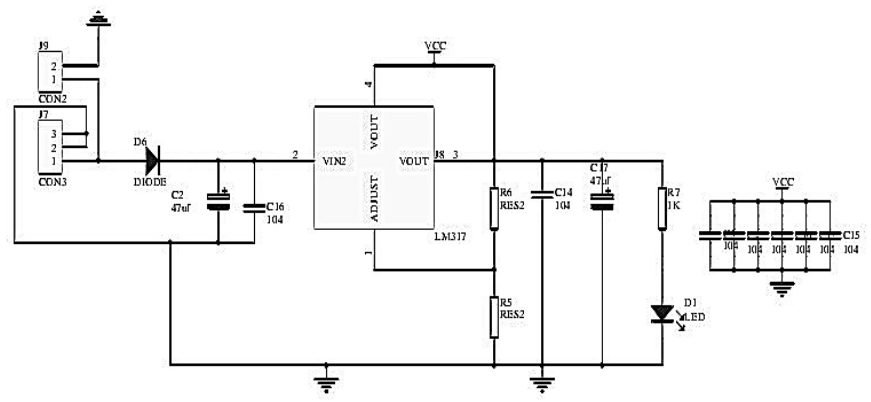

Fig.3

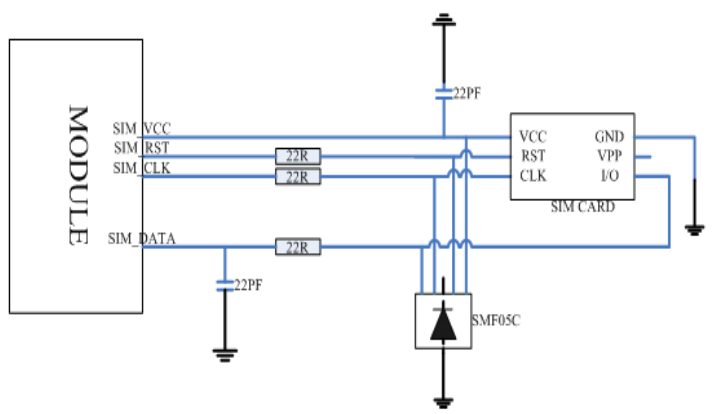

Fig. 4

It can be used with the SIM900A module to share a voltage because the STC11F04E microprocessor supply voltage range of DC $5.5 \mathrm{~V} \sim 3.5 \mathrm{~V}$ wide voltage range.

\section{Design of GPRS module}

Terminal and central server for data transmission needs through wireless communication. Therefore, the terminal need a wireless communication module. This design uses the SIM900A chip of SIMCom company, which power supply voltage: $3.4 \mathrm{~V}-4.5 \mathrm{~V}$.

The design of the SIM card slot is used in 6 PIN card slot and the card slot does not have a SIM card detection function.Circuit using SMF05C as an electrostatic protection device. IO mouth lines in the series resistance of the 22 for matching the impedance between the module and a SIM card and data signal line SIM data have been in the internal module pull.Note that the SIM card peripheral circuit device should be located near the SIM.SIM card circuit wiring diagram 6 pin as shown in Fig. 4. 


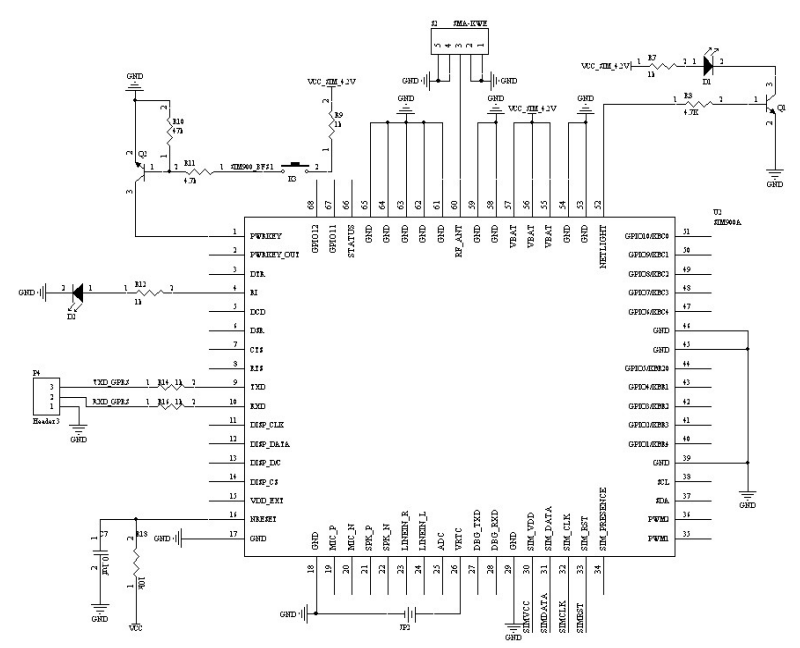

Fig. 5

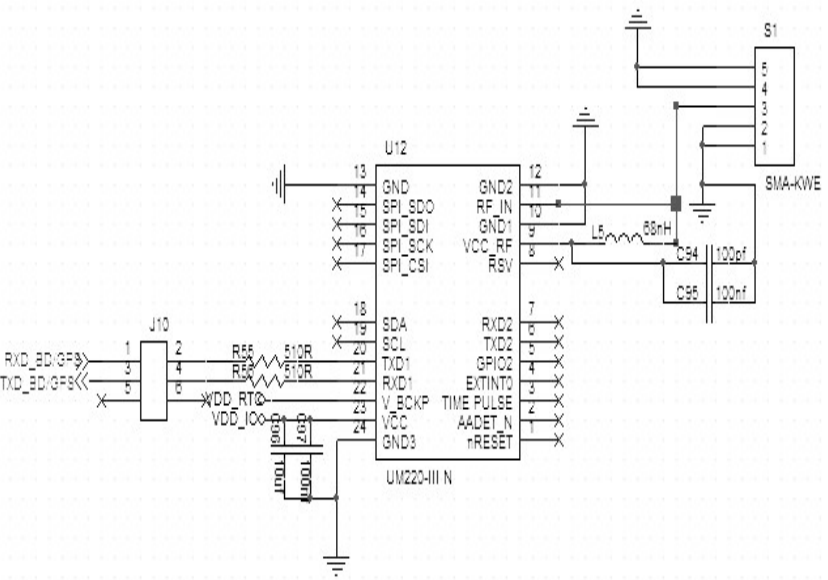

Fig.6

SIM900A main chip circuit design mainly includes the design of switch machine module, RTC power supply design and network status indicator design. The switch machine module uses the PWRKEY pin to open the module, and the PWRKEY signal is pulled for a period of time and then released to boot at this time this pin is pulled up to $3 \mathrm{~V}$.RTC of the module can be used to complete a large capacitor or battery. There is a current limiting resistor in the module, so you can use a single button battery or a large capacitor directly to the VRTC pin to provide power to the RTC.This design uses a button battery directly to the RTC module power supply.The NETLIGHT signal is used to drive the LED lamp.SIM900A main chip design circuit diagram is shown in Fig.5.

\section{BD/GPS module design}

Positioning system is an important part of the vehicle navigation terminal and the design is based on China's independent research and development of satellite positioning system BD Positioning System.

UM220-III-N main chip is designed to provide the $3.3 \mathrm{~V}$ power supply module VCC. The module will be all GND pins and connect the RF_IN signal to the antenna.Note line $50 \mathrm{ohm}$ impedance matching.The main circuit diagram of the chip is shown in Fig.6.

\section{Software design}

\section{Control terminal software design of mobile phone}

This design use map as a development tool, as shown in Fig. 7.Enter the corresponding terminal of the SIM card number in the corresponding prompt box and then click the get button position, so corresponding to the calling procedure may be short message command and send a command to the terminal. The $\mathrm{BD}$ module get latitude and longitude of the current position and then returned to the mobile phone through the short message After receiving the command terminal is activated.Mobile software to extract the corresponding latitude and longitude information printed to the corresponding position on the map to realize tracking of volatile crowd. 


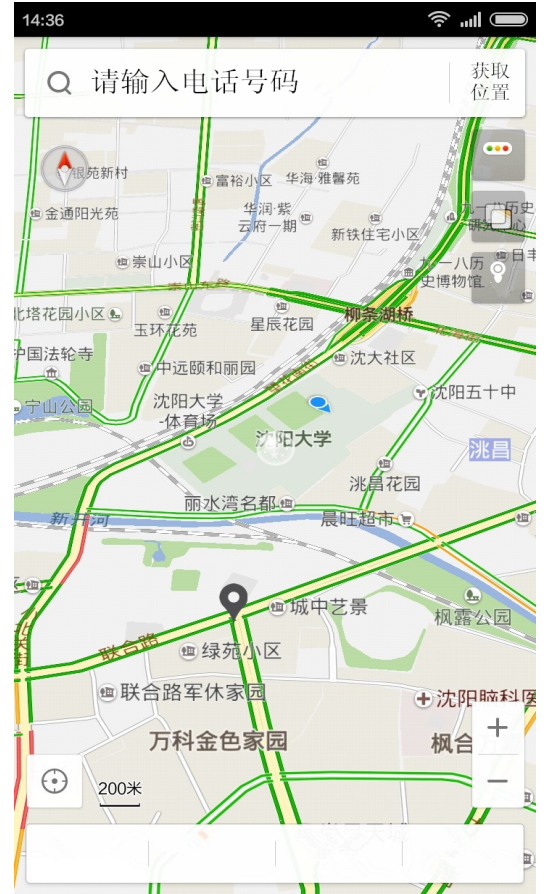

Fig.7

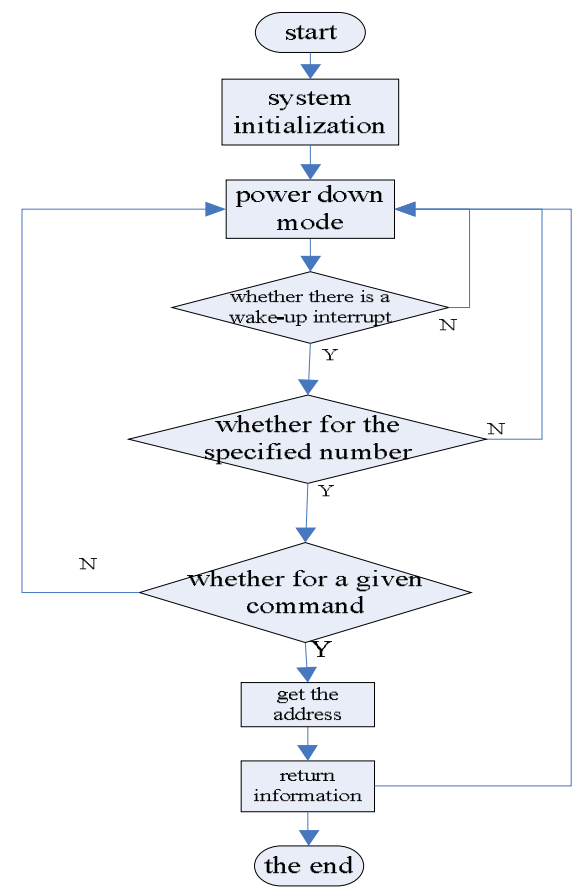

Fig. 8

\section{Terminal software design}

System flow chart is shown in Fig.8.

\section{Conclusion}

The terminal device can achieve location of remote terminal and will obtain location display directly to the phone to control the end of the corresponding map position after debugging and running. The control software based on Android is simple and easy to implement.

\section{References}

[1]Zhou Jia, Li-rong Shen, Wu Jia, Ya-dong Yu. Based on GPS/GSM technology against the loss of personal positioning system design [J]. Journal of electronics,2010,04:39-40+14.

[2]Wang Xiao-nan Xing-bo Zhang, Bing-xiang Li . City bus monitoring device based on GPRS and the base station on [J]. Journal of electronic technology applications, 2013, 11:39-41.

[3]Chen Xiao. BD2/GPS dual-mode vehicle navigation terminal overall design [J]. Journal of management, 2014, 14:383-384.

[4]Zhang Bo, Shuang-ming Zhao. Baidu map based on the Android platform development research [J]. Journal of software Tribune,2015,07:96-99.

[5]Xiao-rong Ren. Single chip microcomputer system reliability design [J]. Computer measurement and control,2003,08:621-623+626. 